\title{
Histories of Neutrino Oscillation of Consistency Induced by the Presence of Normal Matter
}

\author{
Fazeel Khan ${ }^{1, *(1)}$ and Jerzy Dajka ${ }^{1,2, *(1)}$ \\ 1 Institute of Physics, University of Silesia in Katowice, 40-007 Katowice, Poland \\ 2 Silesian Center for Education and Interdisciplinary Research, University of Silesia in Katowice, \\ 41-500 Chorzow, Poland \\ * Correspondence: ahmed.khan@smcebi.edu.pl (F.K.); jerzy.dajka@us.edu.pl (J.D.)
}

Citation: Khan, F.; Dajka, J. Histories of Neutrino Oscillation of

Consistency Induced by the Presence of Normal Matter. Universe 2022, 8, 106. https://doi.org/10.3390/ universe 8020106

Academic Editor: Maria Vasileiou

Received: 18 December 2021

Accepted: 3 February 2022

Published: 6 February 2022

Publisher's Note: MDPI stays neutral with regard to jurisdictional claims in published maps and institutional affiliations.

Copyright: (c) 2022 by the authors. Licensee MDPI, Basel, Switzerland. This article is an open access article distributed under the terms and conditions of the Creative Commons Attribution (CC BY) license (https:// creativecommons.org/licenses/by/ $4.0 /)$.

\begin{abstract}
Oscillation of two-flavour neutrinos is considered within a quantum mechanical framework of consistent (decoherent) dynamic histories. We investigate how consistency of selected three-time histories is affected by oscillation parameters. We show that the presence of normal matter is crucial to maintain consistency of certain classes of neutrinos' dynamic histories and that the consistency does not depend on a Majorana phase and remains insensitive to a potential CP violation.
\end{abstract}

Keywords: neutrino oscillation; consistent histories; CP violation

\section{Introduction}

A measurement allows gaining knowledge about the present of quantum systems. It is, however, difficult to infer from this what the system's past had been. It is even harder to say something about quantum systems that are essentially closed (such as, for example, the whole universe is) and which do not allow for an external observer, or those that are simply resistant to measuring attempts. Neutrinos, hardly interacting with anything, can serve as a natural example of such a 'measurement-resistive' system. Timeevolving neutrinos $[1,2]$ and their oscillations are an example of quantum systems that are particularly difficult to measure. Despite tremendous progress in experimental techniques, even the latest and most sophisticated experiments [3] do not resolve all doubts concerning the fundamental properties of these mysterious and fascinating particles, stimulating research from various perspectives. The dynamic properties of neutrino oscillations [4-6], initially studied in the most natural particle physics [1] context, are rich of various aspects typically related to quantum information processing [5,7-13]. Such a broad and multiperspective interest is motivated not only by the natural applicability of methods and computational techniques borrowed from quantum information processing in the domain of particle physics, but also by recent proposals of information transfer protocols utilizing neutrinos [14] as a resource, or even reflecting the enduring human dream of interstellar communication [15].

The discovery of neutrino oscillations was the first evidence in favour of physics beyond the Standard Model, obviously attracting the particle community [16]. Complementary studies of neutrino oscillations from the perspective of quantum information and communication are naturally supported by an effective description of neutrinos, after limiting to a simplified two-flavour model, as a qubit $[4,10,12,13,17-19]$. The time evolution of a neutrino qubit originates from a fundamental non-correspondence between the neutrino's flavour $\left\{\left|v_{e}\right\rangle,\left|v_{\mu}\right\rangle\right\}$ and massive states $|1\rangle,|2\rangle$. Motivation for, and even the importance of, such research is additionally boosted by results of recent neutrino experiments [3] regarding possible non-trivial $C P$-violations. Potentially violated $C P$ symmetry may indicate a need for the reformulation of many fundamental aspects of the universe from the micro to macro scales. It is because of the charge-conjugation parity reversal that $C P$ symmetry between 
matter and antimatter can be questioned in light of the results of the above-mentioned experiment [3].

Making a measurement and finding a quantum particle in a particular quantum state does not allow inferring its state in the past. Orthodox researchers claim that one cannot talk about a quantum particle between measurements at all. This obvious limitation of standard quantum theory inspires its extensions. The two-state vector formalism [20] and consistent (or decoherent) histories approaches [21-25] are but two fruitful examples going beyond (and sometimes across) the Copenhagen interpretation. We have recently been witnessing how the two above-mentioned approaches are competitively applied to a problem of the past behaviour of a quantum particle in an interferometric experiment [26-29]. Consistent histories, which we further utilize in this paper, are known to gain an additional insight to problems ranging from the small—but fundamental [30-33] — to the largest of scales [34,35]. The consistency of histories allows one to assign probabilities to sequences of suitably defined events for a quantum system beyond that which can be achieved with the Born rule only. As the quantum events in this perspective do not rely on the notion of measurement per se, the consistent histories approach enables one to design a new type of logical approach [22]. This approach is essentially different than the Birkhoff and von Neumann quantum logic [36]. There is a particular practical advantage of using consistent histories formalism to gain information about a system if an external measurement is not available either because of fundamental reasons, or simply technical reasons. Quantum reasoning based on consistent histories [37] uses projective decomposition of identity $P D I=\left\{P^{k}\right\}$, a framework,

$$
P^{j} P^{k}=\delta_{j k} P^{k}, \sum_{j} P^{j}=I
$$

as a cornerstone [21], serving as a quantum counterpart of the event algebra used in standard stochastics. The operators $P_{k} \in P D I$ are projectors mapping quantum states on subspaces of a Hilbert space of the quantum system under analysis. If the subspace is one dimensional, $P_{k}=|k\rangle\langle k|$ where $|k\rangle$ is a vector spanning the subspace. Projectors $P_{k} \in P D E$ correspond to quantum properties i.e., features of a quantum system. Contrary to standard quantum logic [36], in the consistent histories approach, logical operations, such as $k_{1} \wedge k_{2}$, can be applied to the quantum properties $k_{1,2}$ provided that the properties are compatible, i.e., they correspond to commutative projectors $\left[P_{k_{1}}, P_{k_{2}}\right]=0$ with an obvious relation to the co-measurability of the observables in 'usual' quantum mechanics. PDIs equipped with the admissible logical operation are (for the consistent histories approach) natural analogues of event algebras in standard stochastics.

A quantum history (an analogue of a random process) of length $n$ of a quantum system is a sequence of quantum events at successive times $\left\{P_{i}\right\}$, with one projector for each time $t_{i}, i=0, \ldots, f$. There is, however, one fundamental additional requirement imposed: it is the single framework rule [21,30,37] bounding the meaningfulness of simultaneous reasoning to physical properties that are compatible [21,30,31,37]. In other words, one cannot formulate meaningful statements concerning the properties of observables unless they are co-measureable. In particular, one can assign probabilities to events provided that they belong to a single framework. This requirement imposed by the consistent histories approach allows one to dissolve otherwise paradoxical features [30,37]. At each time instant, $t_{i}$, all the quantum properties of a system correspond to elements of a $\operatorname{PDI}\left(t_{i}\right)$. The properties have their assigned probabilities and a time evolution of the quantum system studied with consistent histories model can be considered as a stochastic process. With such an approach, neither the future nor the past states of the system must be determined by the present state. Instead, they are (only) related by the probabilities approaching particular 
values which are zero or one for a deterministic time evolution. A mathematical way to describe sequences of events is the tensor product of 'instantaneous' Hilbert spaces $\mathcal{H}\left(t_{i}\right)$

$$
\breve{\mathcal{H}}=\bigodot_{i=0}^{n} \mathcal{H}\left(t_{i}\right)
$$

where, following [21], we applied $\odot$ instead of $\otimes$ as a symbol for temporal (multi-time) rather than spatial (multi-partite) composites. Let us note, however, that in most cases (and, in particular, further in this work) the time dependence of a system's Hilbert space is trivial i.e., $\mathcal{H}\left(t_{i}\right)=\mathcal{H}$ for $i=0, \ldots, n$. In formal terms, quantum properties (events) related to time-evolving systems are its histories forming time-dependent PDI

$$
\begin{aligned}
M^{j} M^{k} & =\delta_{j k} M^{k}, \text { where } \sum_{j} M^{j}=\bigodot_{i=0}^{n} I=\breve{I} \in \mathcal{B}(\breve{\mathcal{H}}) \\
M^{k} & =\bigodot_{i=0}^{n} P^{(i, k)}, \quad P^{(i, k)} \in \operatorname{PDI}\left(t_{i}\right)
\end{aligned}
$$

where (generically) $\operatorname{PDI}\left(t_{i}\right) \neq \operatorname{PDI}\left(t_{j}\right)$ for $j \neq i$. Then, there is a chance to pose different questions concerning different quantum properties of the systems at different time instants. Assigning probabilities to non-commuting quantum properties in Equation (3) is meaningful only if there is no interference between pairs of histories that are supposed to be decoherent. After extending the celebrated Born rule to multi-time history $M^{i}$ (the consistent histories formalism exhibits, here, its true predictive power), one can assign it a weight [21,22,30,31,37]:

$$
W\left(M^{k}\right)=\left\langle K\left(M^{k}\right), K\left(M^{k}\right)\right\rangle=\operatorname{Tr}\left[K^{\dagger}\left(M^{k}\right) K\left(M^{k}\right)\right]
$$

where for a time evolution operator $T\left(t_{i}, t_{j}\right)$

$$
K\left(M^{k}\right)=P^{(n, k)} \prod_{i=0}^{n-1} T\left(t_{i+1}, t_{i}\right) P^{(i, k)}=P_{n} T\left(t_{n}, t_{n_{1}}\right) P_{n-1} \cdots P_{1} T\left(t_{1}, t_{0}\right) P_{0}
$$

is termed as the chain operator [21]. Then the consistency condition [21,22,30,31,37] granting the decoherence of histories, reads as follows:

$$
\left\langle K\left(M^{k}\right), K\left(M^{s}\right)\right\rangle=0, \text { for all } k \neq s
$$

The rationale behind Equation (6) [21] is rooted in standard probability theory. As the histories Equation (3) form a $P D I$, the meaningful linear combinations $M=\sum_{i} c_{i} M^{i}$ can also be constructed. The corresponding weight reads as follows:

$$
W(M)=\sum_{i} c_{i} W\left(M^{i}\right)
$$

On the other hand, the linearity of $K(M)=\sum_{i} c_{i} K\left(M^{i}\right)$ Equation (4) implies

$$
W(M)=\sum_{i} \sum_{j} c_{i} c_{j}\left\langle K\left(M^{i}\right), K\left(M^{j}\right)\right\rangle
$$

Let us note that Equations (7) and (8) can be satisfied provided that Equation (6) is granted.

Let us note that a quantum history of a physical system is a sequence of quantum events at successive times, where a quantum event at a particular time can be any quantum property of the system in question [21]. Such a convenient tool can serve to analyse properties of systems that are very difficult to measure or replicate experimentally, as in 
the case of neutrinos. A neutrino in a particular mass state is an example of 'an event' of a quantum property that is hard (maybe technically impossible) to measure. It is because the neutrino masses corresponding to different mass states are extremely small, and probably too small to resolve experimentally. That is why one is left with considering coherent superposition of the mass states: the flavour states. As a result, one recognizes two classes of neutrino oscillation experiments. In the first, having a beam of a given flavour, one may investigate how many neutrinos have disappeared. In the second, starting with a pure beam of a particular and known flavour, one may investigate how often neutrinos of a different flavour are detected [2].

In this paper, we consider a simplified two-flavour model of neutrino oscillation limited to the electron-muon neutrino oscillation $[1,2,4,17,18]$. Despite the simplicity of the two-flavor model (as it is analytically treatable), it allows one to understand some of the origins and mechanisms behind the oscillations. We also consider the shortest nontrivial three-time histories of the two-flavour neutrino evolution. Such histories cannot be analysed solely within standard Born rule and the formalism of consistent histories allows one to investigate their properties inaccessible from the standard treatment. The beginning and ending points of the histories are events (in the sense described above) of a neutrino in a flavour state. However, as the intermediate event of the three-time history, there is a neutrino in a mass state. According to the decoherent histories model [21], such histories, provided that they are consistent, allow inferring otherwise non-measured, but in principle measurable, quantum properties of the oscillating neutrino. Our primary aim is to investigate consistency of such histories induced by the presence of normal matter affecting the oscillation. We show that modification of neutrino dynamics due to this presence of matter makes the histories otherwise belonging to inconsistent family of dynamical events consistent. We show that a potential CP-violation (indicated by non-vanishing Majorana phase) does not affect the consistency of the considered neutrino histories, and, hence, our findings hold true regardless of neutrino being a Dirac or Majorana particle. We show that the presence of normal matter is a feature that plays a crucial role in the dynamic properties and consistency of the neutrino oscillation considered here.

\section{Histories of Two-Flavour Neutrino Oscillation}

Working in a two-flavour approximation, we consider two orthogonal vacuummassive states, $|1\rangle$ and $|2\rangle$, and two neutrino flavours: electron $(e)$ and muon $(\mu)$. The electron neutrino state is given by $\left|v_{e}\right\rangle=\cos \theta|1\rangle+e^{i \phi} \sin \theta|2\rangle$, and the muon neutrino state is $\left|v_{\mu}\right\rangle=-\sin \theta|1\rangle+e^{i \phi} \cos \theta|2\rangle$, where $\theta=\theta_{12}$ is the mixing angle. The phase $\phi$ is the Majorana phase (CP violating), which is non-zero under the U(1) global transformation in the Majorana case, unlike that in the Dirac case, where this transformation renders it zero, or eliminates it [1]. A vacuum Hamiltonian-generating oscillation of a relativistic neutrino [1] is given by

$$
H_{0}=\left(\begin{array}{cc}
E-\frac{\Delta m_{21}^{2}}{4 E} & 0 \\
0 & E+\frac{\Delta m_{21}^{2}}{4 E}
\end{array}\right)
$$

where $\Delta m_{21}^{2}=m_{2}^{2}-m_{1}^{2}$ is the square-mass splitting of the mass states in the normal hierarchy case and $E$ is the energy of the massless neutrino. The neutrino Wolfenstein effective Hamiltonian [1,4] while propagating in matter is given by

$$
H=H_{0}+\frac{V_{0}}{2}\left(\begin{array}{cc}
1+\cos 2 \theta & e^{-i \phi} \sin 2 \theta \\
e^{i \phi} \sin 2 \theta & 1-\cos 2 \theta
\end{array}\right)
$$

where $V_{0}=\sqrt{2} \cos ^{2} \theta_{13} G_{F} N_{e}, 0.953 \leq \theta_{13} \leq 1$ [1] is one of the oscillation parameters with $3 \sigma$ bound, $N_{e}$ is the electron density and $G_{F}$ is the Fermi constant. $V_{0}$ is the effective interaction potential due to oscillation of the neutrino in the presence of ordinary matter. It results from the coherent forward scattering on electrons via charged current interactions [1]. 
The corresponding charged current interaction term for muon is not included, as only the usual matter is considered. As per the standard model, the neutral current interaction does not enter effectively into the Hamiltonian [1].

Information gained from applying the consistent history approach to quantum dynamics becomes non-trivial if one considers at least three-time histories. Such histories cannot be directly analyzed with the Born rule [21]. A family of three-times histories of quantum events at $t_{0}, t_{1}$ and $t_{2}$ that is going to be considered, here, is the following:

$$
\begin{aligned}
& M^{0}:\left[v_{\mu}\right] \odot[I] \odot[I] \\
& M^{1}:\left[v_{e}\right] \odot[1] \odot\left[v_{\mu}\right] \\
& M^{2}:\left[v_{e}\right] \odot[1] \odot\left[v_{e}\right] \\
& M^{3}:\left[v_{e}\right] \odot[2] \odot\left[v_{\mu}\right] \\
& M^{4}:\left[v_{e}\right] \odot[2] \odot\left[v_{e}\right]
\end{aligned}
$$

where $[x]=|x\rangle\langle x|, x=v_{e, \mu}, 1,2$. As discussed in the previous section, the presence of $[x=1]$ or $[x=2]$ as a middle event in the histories corresponds to a mass state as neutrino's quantum property. All the histories in Equation (11), except $M^{0}$, which is included to obtain a temporal $P D I$, describe a neutrino starting in an electron flavour and finishing in one of two flavours: muon or electron. Our objective is to verify if one can consistently include a quantum event $[i], i=1,2$ in the oscillation history i.e., whether the neutrino, being in its mass state, is an event of a consistent ontological value [31]. The family Equation (11) describes different possibilities (histories) starting from an electron neutrino only, but an analogous family starting with a muon neutrino can be defined and all the following results remain valid for such a choice. The chain operators

$$
\begin{aligned}
& K\left(M^{0}\right)=T_{2} T_{1}\left|v_{\mu}\right\rangle\left\langle v_{\mu}\right| \\
& K\left(M^{1}\right)=\left\langle v_{\mu}\left|T_{2}\right| 1\right\rangle\left\langle 1\left|T_{1}\right| v_{e}\right\rangle \cdot\left|v_{\mu}\right\rangle\left\langle v_{e}\right| \\
& K\left(M^{2}\right)=\left\langle v_{e}\left|T_{2}\right| 1\right\rangle\left\langle 1\left|T_{1}\right| v_{e}\right\rangle \cdot\left|v_{e}\right\rangle\left\langle v_{e}\right| \\
& K\left(M^{3}\right)=\left\langle v_{\mu}\left|T_{2}\right| 2\right\rangle\left\langle 2\left|T_{1}\right| v_{e}\right\rangle \cdot\left|v_{\mu}\right\rangle\left\langle v_{e}\right| \\
& K\left(M^{4}\right)=\left\langle v_{e}\left|T_{2}\right| 2\right\rangle\left\langle 2\left|T_{1}\right| v_{e}\right\rangle \cdot\left|v_{e}\right\rangle\left\langle v_{e}\right|
\end{aligned}
$$

grant consistency of the corresponding histories provided that $\left\langle K\left(M^{i}\right) \mid K\left(M^{j}\right)\right\rangle=$ 0 for all $i \neq j$. Applying the consistency condition to all members of the family, we see that all histories are mutually exclusive of each other, regardless of the dynamics of the system apart from $M^{1}$ versus $M^{3}$, and $M^{2}$ versus $M^{4}$. We have

$$
\begin{aligned}
\left\langle K\left(M^{1}\right) \mid K\left(M^{3}\right)\right\rangle & =\left\langle 1\left|T_{2}^{\dagger}\right| v_{\mu}\right\rangle\left\langle v_{e}\left|T_{1}^{\dagger}\right| 1\right\rangle\left\langle v_{\mu}\left|T_{2}\right| 2\right\rangle\left\langle 2\left|T_{1}\right| v_{e}\right\rangle \\
\left\langle K\left(M^{2}\right) \mid K\left(M^{4}\right)\right\rangle & =\left\langle 1\left|T_{2}^{\dagger}\right| v_{e}\right\rangle\left\langle v_{e}\left|T_{1}^{\dagger}\right| 1\right\rangle\left\langle v_{e}\left|T_{2}\right| 2\right\rangle\left\langle 2\left|T_{1}\right| v_{e}\right\rangle
\end{aligned}
$$

where $T_{1}=\exp \left[i\left(t_{0}-t_{1}\right) H\right]$ and $T_{2}=\exp \left[i\left(t_{1}-t_{2}\right) H\right]$ represent the time evolution operators for the first and second interval of the three-time histories Equation (11). The aim is to recognize conditions turning the family of histories Equation (11) consistent:

$$
\begin{aligned}
\left\langle K\left(M^{1}\right) \mid K\left(M^{3}\right)\right\rangle & \equiv 0 \\
\left\langle K\left(M^{2}\right) \mid K\left(M^{4}\right)\right\rangle & \equiv 0
\end{aligned}
$$

\subsection{Vacuum-Only Oscillation}

Consistent histories approach inherently depends on the probability distribution used to assign probabilities of quantum events, and, thus, on the dynamics of the system [21]. For further simplicity, we set initial time $t=0$ and we compute the inner products between the chain operators for $M^{1}$ and $M^{3}$, and between $M^{2}$ and $M^{4}$ Equation (13) to equate them 
to zero, and obtain the consistency Equation (14). To achieve the consistency Equation (14) in vacuum (i.e., for the time evolution generated by $H=H_{0}$ Equation (9)), at least one of the three equations that follow need to be satisfied:

$$
\begin{array}{r}
\cos \left(t\left(E-\frac{\Delta m_{21}^{2}}{4 E}\right)\right)-i \sin \left(t\left(E-\frac{\Delta m_{21}^{2}}{4 E}\right)\right)=0 \\
\cos \left(t\left(E+\frac{\Delta m_{21}^{2}}{4 E}+\frac{\phi}{t}\right)\right)-i \sin \left(t\left(E+\frac{\Delta m_{21}^{2}}{4 E}+\frac{\phi}{t}\right)\right)=0 \\
\cos \left(t\left(E+\frac{\Delta m_{21}^{2}}{4 E}-\frac{\phi}{t}\right)\right)-i \sin \left(t\left(E+\frac{\Delta m_{21}^{2}}{4 E}-\frac{\phi}{t}\right)\right)=0
\end{array}
$$

where $t$ is either $t_{1}$, or $t_{2}$ representing time intervals between the first and second events in the histories Equation (13). Let us note that either the real parts in Equation (15) can be zeroed, or the imaginary parts, but not both simultaneously. Thus, in the absence of matter, the family of histories Equation (11) is never consistent under the dynamics generated by $H_{0}$ in Equation (9). In other words, in the absence of matter, one cannot credibly assign consistency, and, hence, probability, to the events in Equation (11). It changes, however, in the presence of matter.

\subsection{The Impact of Matter}

Let us now consider a neutrino propagating in matter. The effective Hamiltonian describing two-flavour neutrino oscillation in the presence of normal matter Equation (10), written in its diagonal representation, reads as follows:

$$
H=\frac{1}{2}(A+B+D)|X\rangle\left\langle X\left|+\frac{1}{2}(A+B-D)\right| Y\right\rangle\langle Y|
$$

where

$$
\begin{aligned}
& A=E-\frac{\Delta m_{21}^{2}}{4 E}+\frac{V_{0}}{2}(1+\cos 2 \theta) \\
& B=E+\frac{\Delta m_{21}^{2}}{4 E}+\frac{V_{0}}{2}(1-\cos 2 \theta) \\
& C=\frac{V_{0}}{2} \sin 2 \theta \\
& D=\sqrt{(A-B)^{2}+4 C^{2}}
\end{aligned}
$$

and the Eigenstates:

$$
\begin{aligned}
& |X\rangle=\frac{1}{u}\left(|1\rangle+\frac{1}{2} \frac{e^{i \phi}(B-A+D)}{C}|2\rangle\right), u=\frac{\sqrt{4 C^{2}+e^{i 2 \phi}(B-A+D)^{2}}}{2 C} \\
& |Y\rangle=\frac{1}{v}\left(|1\rangle+\frac{1}{2} \frac{e^{i \phi}(B-A-D)}{C}|2\rangle\right), v=\frac{\sqrt{4 C^{2}+e^{i 2 \phi}(B-A-D)^{2}}}{2 C}
\end{aligned}
$$

The flavour states expressed in terms of the Eigenstates in Equation (18) read as:

$$
\begin{aligned}
& \left|v_{e}\right\rangle=\frac{1}{2 D}[u J|X\rangle-v K|Y\rangle] \\
& \left|v_{\mu}\right\rangle=\frac{1}{2 D}[u L|X\rangle+v N|Y\rangle]
\end{aligned}
$$


where, for further convenience, we introduce

$$
\begin{aligned}
J & =\cos (\theta)(A-B+D)+2 \sin (\theta) C \\
K & =\cos (\theta)(A-B-D)+2 \sin (\theta) C \\
L & =2 \cos (\theta) C-\sin (\theta)(A-B+D) \\
N & =\sin (\theta)(A-B-D)-2 \cos (\theta) C
\end{aligned}
$$

We then compute the inner products between the chain operators for $M^{1}$ and $M^{3}$, and between $M^{2}$ and $M^{4}$, respectively, to the equate them to zero in order to achieve the consistency Equation (14). Our aim is to find values of $V_{0}$ (and possibly $\phi$ ) causing the family of histories Equation (11) consistently.

Let us note that the inner products in Equation (13) consist of four components each. To achieve the said consistency Equation (14), one expects at least one of the components of each inner product to equal zero. Note that unless $t_{1}=t_{2}$, only one of two components in Equation (13) can vanish. However, the particular value of time instants in the evolution providing consistency of the oscillation's history affects only the probability of the histories, but not the consistency itself. Thus, for the sake of computing, it is not necessary to consider the consistency achieved via time evolution at $t_{1}$ or $t_{2}$ separately. It is enough to observe that at least one of the four equations below need to be satisfied:

$$
\begin{aligned}
|u|^{2} L(A-B+D)-|v|^{2} N(A-B-D) e^{i t D} & =0 \\
|u|^{2} J(A-B+D)+|v|^{2} K(A-B-D) e^{i t D} & =0 \\
|u|^{2} L-|v|^{2} N e^{i t D} & =0 \\
|u|^{2} J+|v|^{2} K e^{i t D} & =0
\end{aligned}
$$

All the parameters above, except $u$ and $v$ (having non-trivial imaginary parts), are real. Thus, the inner products Equation (13)) leading to the consistency Equation (14) can be broken down into real and imaginary parts, which are further considered separately. The requirement of the vanishing of the imaginary parts in Equation (21) reads as follows:

$$
\begin{aligned}
|v|^{2} N(A-B-D) \sin (t D) & =0 \\
|v|^{2} K(A-B-D) \sin (t D) & =0 \\
|v|^{2} N \sin (t D) & =0 \\
|v|^{2} K \sin (t D) & =0
\end{aligned}
$$

Let us note that each element of Equation (22) is of a form $|v|^{2} \alpha \gamma \sin (t D)$ where $\alpha$ is either $N$ or $K$ and $\gamma$ is either $(A-B-D)$ or 1 . Clearly, neither $v=0$ as given in Equation (19) nor $\gamma=0$, at least in the presence of normal matter $V_{0} \neq 0$. To see that $\alpha$ cannot be zero requires a little more complicated analysis. If $\alpha=0$ the corresponding real part for each element is of the form $|u|^{2} \beta \delta$, where $\beta$ is either $L$ or $J$ and $\delta$ is either $(A-B+D)$ or 1 . Similar to $v$ and $(A-B-D),|u|^{2}$ and $(A-B+D)$ cannot equal 0 , so $\delta$ cannot be 0 . The only way to achieve consistency, then, is $\beta=0$. As $L$ with $N$ and $J$ with $K$ appear in pairs, both $\alpha$ and $\beta$ being zero would lead to vanishing and, thus, nonphysical flavour states. The imaginary parts in Equation (22) vanish, provided that $\sin (t D)=0$ where $t$ is either $t_{1}$ or $t_{2}$, i.e., provided that $D=\frac{n \pi}{t_{1}}$ or $D=\frac{n \pi}{t_{2}}$ (for $n \in \mathbb{N}$ ) where $t_{1}$, and $t_{2}$ are the time intervals in the set of three-time histories Equation (11). The real parts of Equations (21) should also vanish to allow consistency of histories Equation (11) 


$$
\begin{array}{r}
|u|^{2} L(A-B+D)-|v|^{2} N(A-B-D) \cos (t D)=0 \\
|u|^{2} J(A-B+D)+|v|^{2} K(A-B-D) \cos (t D)=0 \\
|u|^{2} L-|v|^{2} N \cos (t D)=0 \\
|u|^{2} J+|v|^{2} K \cos (t D)=0
\end{array}
$$

Substituting explicit forms of $|u|^{2}$, and $|v|^{2}$ Equations (19)-(23) leads to

$$
\begin{aligned}
(B-A+D) L(A-B+D)+(B-A-D) N(A-B-D) \cos (t D) & =0 \\
(B-A+D) J(A-B+D)-(B-A-D) K(A-B-D) \cos (t D) & =0 \\
(B-A+D) L+(B-A-D) N \cos (t D) & =0 \\
(B-A+D) J-(B-A-D) K \cos (t D) & =0
\end{aligned}
$$

and one concludes that the consistency of the set of histories Equation (11) of neutrinos propagating in matter does not dependent on $\phi$. As vanishing of the imaginary parts of Equation (21) given in Equation (22) holds true provided that $t D=n \pi$ where $n \in \mathbb{N}$, searching for the consistency conditions of Equation (14) can be further split into two cases: (i) for $n$ even and (ii) odd. For $n$ even (i) each of Equations (23) becomes solved for $D=0$, which we reject as nonphysical. Therefore, histories in Equation (11) of our system are inconsistent for $n$ even. On the other hand, for $n$ odd (ii) the (non-negative) solution to the equations Equation (23) is given by $V_{0}=\frac{\Delta m_{21}^{2}}{2 E}$. It imposes the consistency conditions, $V_{0}=\frac{\Delta m_{21}^{2}}{2 E}, D=2 \sin \theta \frac{\Delta m_{21}^{2}}{2 E}$, jointly with $n=\frac{t D}{\pi}$ being an odd number.

\section{Discussion}

Let us place emphasis on a crucial qualitative difference [21] between histories of vanishing probability and inconsistent families of histories. There are non-trivial histories, $M$, for which $K(M)=0$. Such histories are of zero weight, or vanishing probability, and-as they never occur-they are termed as dynamically impossible. On the other hand, inconsistent families of histories are meaningless in the sense that there is no way (at least within consistent history model) to assign probabilities to these histories using time development governed by the laws of quantum dynamics [21]. One might be tempted to say that an inconsistent family of histories never occurs. However, that would be a highly inaccurate description of the physically deep notion of inconsistency [31].

As it is stated earlier in this work, if evolution times $t_{1,2}$ are not equal $t_{1} \neq t_{2}$ and $D$ is either $\frac{n \pi}{t_{1}}$, or $\frac{n \pi}{t_{2}}$ the family of histories Equation (11) is consistent for the dynamics generated by Equation (10). Let us note, however, that a particular choice of $D$ (among $D$ being $\frac{n \pi}{t_{1}}$, or $\frac{n \pi}{t_{2}}$ ) does significantly affect probabilities (weights) of the histories Equation (12). For $n$ odd, $D=2 \sin \theta \frac{\Delta m_{21}^{2}}{2 E}=\frac{n \pi}{t_{1}}$ and $V_{0}=\frac{\Delta m_{21}^{2}}{2 E}$ the family of histories Equation (11) is consistent. This leads to the chain operators $K\left(M^{i}\right)=0, i=1,2$. Thus, the probability of both histories $M^{1}$, and $M^{2}$ in Equation (11) vanishes. The only non-zero histories in the family are then $M^{0,3,4}$. For $D=\frac{n \pi}{t_{2}}$ the consistency of Equation (11) is provided with the chain operators $K\left(M^{i}\right)=0$ for $i=2,3$, i.e., probabilities of histories $M^{2}$ and $M^{3}$ in Equation (11) vanish and the only non-zero histories are then $M^{0,1,4}$. For $t_{1}=t_{2}=t$ and $D=\frac{n \pi}{t}$ the family of histories Equation (11) is consistent with $K\left(M^{i}\right)=0$ for $i=1,2,3$ and the only non-zero histories are $M^{0,4}$.

The results based upon consistent histories presented here confirm, from a qualitative perspective, the Mikheyev-Smirnov-Wolfenstein conditions. The MSW effect not only shows us that the oscillations for neutrinos propagating in matter differ from those propagating in vacuum, but also provides us with the MSW resonance condition for a maximal probability for change of flavor for neutrino. However, using consistent histories approach as we do, devoid of predicting magnitude of the survival or flavour change probability, we rather aim to search for consistency for a complete family of histories where survival or 
change of flavour are both considered with an oscillation to a pure mass state in between. That is why the formalism of consistent histories applied here allows one to investigate the features of the neutrino oscillation problem complementary to the well-established and known results.

\section{Conclusions}

The consistent histories approach allows one to describe quantum time development as a process that is essentially and always stochastic or probabilistic, and not just when measurements are being carried out. The consistency condition allows one to decide which among the potential histories can occur or can even be considered. We investigated if a class of simple, three-time histories Equation (11) (including quantum property of a neutrino having a definite mass) can be consistent. As a result, we infer whether one can assign a probability to such an 'event'.

Our analysis showed that, for an overwhelming majority of cases, the set of histories that we considered remained inconsistent and, as a consequence, meaningless. The family of histories we proposed was inconsistent when a neutrino propagated in vacuum. However, under certain tailored dynamical conditions, the family of histories became consistent when a neutrino propagated in the presence of matter, provided that an interaction potential, $V_{0}$, took a very particular value. This value was not only precisely determined by the consistent histories formalism, but also needed to be confronted with known experimental estimates reading for solar neutrinos $\Delta m_{21}^{2}=1.4906 \times 10^{-60} m_{P}\left(8 \times 10^{-5} \mathrm{eV}^{2}\right)$ [38].

The magnitudes of $V_{0}$ and $D$ were extremely small for even a relatively meager value of $E$ for our analysis. We recalled that the quantity $D$ represents twice the difference in Eigenvalues for our system and, for such small values of $D$, the difference in the Eigenvalues for the system was also extremely small. From this perspective, consistent histories provided additional and supplementary evidence for the neutrino mass states' being hardly accessible. This result was gained not only from technical viewpoint, as it is in the 'usual' quantum mechanics, but also from a fundamental perspective, as it was enforced by the consistent histories approach. Although the Majorana $C P$-violation phase does not influence the neutrino oscillation in a 2-flavour case [39], consistency conditions could shed light on neutrinos being Dirac or Majorana type if consistency would depend on it. The consistency of the class of histories investigated in this paper was not affected by the $C P$-violating Majorana phase; however, the results presented here, then, hold true for both Majorana or Dirac neutrinos, and would be unchanged after the $C P$ problem becomes (sooner or later) finally resolved.

In our work, we aimed to present the general properties that lead to the consistency of the proposed family of histories in a general setting for a matter-assisted propagation, not limited to a particular type of neutrino. In our model calculations, we showed that normal matter plays a crucial role in inducing the consistency of certain classes of histories of neutriono oscillations. It suggests that considering vacuum-only models may be, at least for some purposes, an oversimplification. Such an oversimplification may result in excludingas seemingly meaningless-quantum properties that otherwise can, at least in principle and in a tailored parameter regime, be consistently studied.

Quantum formalism based on consistent histories is of great predictive power, as it can be applied to gain a useful knowledge concerning quantum systems without measuring them. There are quantitative properties that can be calculated using consistent formalism and are inaccessible in a standard quantum approach. Probabilities (weights) of multi-time histories going beyond a standard Born rule are probably the best examples thereof. On the other hand, consistent histories, such as a quantum formalism, allow one to investigate the qualitative features of quantum evolution, such as the consistency of certain histories. As considering the consistency, per se, goes beyond standard quantum formalism, all the results obtained in our work are essentially novel with respect to that known about neutrino oscillation so far. The qualitative results concerning the consistency of certain histories presented in our work were supplemented with quantitative predictions on consistency- 
granting parameters. Let us emphasise that, at least in our work, a primary advantage is using consistent histories as a predictive tools for the qualitative properties of neutrino oscillations. We hope that our contribution may motivate further studies of neutrino properties with consistent histories' formalism as a useful complementary tool.

Author Contributions: Calculation, F.K.; Investigation, F.K. and J.D.; Writing-Review and Editing, F.K. and J.D. All authors have read and agreed to the published version of the manuscript.

Funding: This research received no external funding.

Data Availability Statement: Not applicable.

Conflicts of Interest: The authors declare no conflict of interest.

\section{References}

1. Giunti, C.; Wook, K.C. Fundamentals of Neutrino Physics and Astrophysics; Oxford University: Oxford, UK, 2007.

2. Fantini, G.; GalloRosso, A.; Zema, V.; Vissani, F. Introduction to the Formalism of Neutrino Oscillations. In The State of the Art of Neutrino Physics; 2018 ; Chapter 2, pp. 37-119. Available online: https://www.worldscientific.com/doi/pdf/10.1142/9789813226 098_0002 (accessed on 1 December 2021 ). [CrossRef]

3. The T2K Collaboration. Constraint on the matter-antimatter symmetry-violating phase in neutrino oscillations. Nature 2020, 580, 339-344. [CrossRef] [PubMed]

4. $\quad$ Benatti, F.; Floreanini, R. Massless neutrino oscillations. Phys. Rev. D 2001, 64, 085015. [CrossRef]

5. Banerjee, S.; Alok, A.K.; Srikanth, R.; Hiesmayr, B.C. A quantum information theoretic analysis of three flavor neutrino oscillations. Eur. Phys. J. 2015, C75, 487. [CrossRef] [PubMed]

6. Molfetta, G.D.; Pérez, A. Quantum walks as simulators of neutrino oscillations in a vacuum and matter. New J. Phys. 2016, 18, 103038. [CrossRef]

7. Gangopadhyay, D.; Home, D.; Roy, A.S. Probing the Leggett-Garg Inequality for Oscillating Neutral Kaons and Neutrinos. Phys. Rev. 2013, A88, 022115. [CrossRef]

8. Alok, A.K.; Banerjee, S.; Sankar, S.U. Quantum correlations in terms of neutrino oscillation probabilities. Nucl. Phys. 2016, B909, 65-72. [CrossRef]

9. Blasone, M.; Dell'Anno, F.; Siena, S.D.; Illuminati, F. Entanglement in neutrino oscillations. EPL Europhys. Lett. 2009 , 85, 50002. [CrossRef]

10. Richter, M.; Dziewit, B.; Dajka, J. Leggett-Garg $K_{3}$ quantity discriminates between Dirac and Majorana neutrinos. Phys. Rev. D. 2017, 96, 076008. [CrossRef]

11. Antonio, C.; Salvatore, M.G.; Gaetano, L.; Quaranta, A. Discerning the Nature of Neutrinos: Decoherence and Geometric Phases. Universe 2020, 6, 207. [CrossRef]

12. Khan, F.; Dajka, J. Geometric speed limit of neutrino oscillation. Quantum Inf. Process. 2021, 20, 193. [CrossRef]

13. Khan, F.; Dajka, J. Neutrino Oscillations in the Presence of Matter and Continuous Non-Selective Measurement. Symmetry 2020, 12, 1296. [CrossRef]

14. Stancil, D.D.; Adamson, P.; Alania, M.; Aliaga, L.; Andrews, M.; Del Castillo, C.A.; Bagby, L.; Bazo Alba, J.L.; Bodek, A.; Boehnlein, D.; et al. Demonstration Communication Using Neutrinos. Mod. Phys. Lett. A 2012, 27, 1250077. [CrossRef]

15. Liu, C. The Dark Forest (Remembrance of Earth's Past, 2); Tom Doherty Associates Book: New York, NY, USA, 2008.

16. Bilenky, S. Neutrino oscillations: From a historical perspective to the present status. Nucl. Phys. B 2016, 908, 2-13. [CrossRef]

17. Benatti, F.; Floreanini, R. Open Quantum Dynamics: Complete Positivity and Entanglement. Int. J. Mod. Phys. B 2005, 19, 3063-3139. [CrossRef]

18. Dajka, J.; Syska, J.; Luczka, J. Geometric phase of neutrino propagating through dissipative matter. Phys. Rev. D 2011, 83, 097302. [CrossRef]

19. Richter-Laskowska, M.; Łobejko, M.; Dajka, J. Quantum contextuality of a single neutrino under interactions with matter. New J. Phys. 2018, 20, 063040. [CrossRef]

20. Aharonov, Y.; Vaidman, L. The Two-State Vector Formalism: An Updated Review. In Time in Quantum Mechanics; Muga, J., Mayato, R.S., Egusquiza, Í., Eds.; Springer: Berlin/Heidelberg, Germany, 2008; pp. 399-447. [CrossRef]

21. Griffiths, R.B. Consistent Quantum Theory; Cambridge University Press: Cambridge, UK, 2003.

22. Griffiths, R.B. Consistent histories and the interpretation of quantum mechanics. J. Stat. Phys. 1984, 36, 219-272. [CrossRef]

23. Omnès, R. Logical reformulation of quantum mechanics. I. Foundations. J. Stat. Phys. 1988, 53, 893-932. [CrossRef]

24. Omnès, R. The Interpretation of Quantum Mechanics; Princeton University Press: Princeton, NJ, USA, 1994.

25. Gell-Mann, M.; Hartle, J.B. Classical equations for quantum systems. Phys. Rev. D 1993, 47, 3345-3382. [CrossRef]

26. Vaidman, L. Past of a quantum particle. Phys. Rev. A 2013, 87, 052104. [CrossRef]

27. Griffiths, R.B. Particle path through a nested Mach-Zehnder interferometer. Phys. Rev. A 2016, 94, 032115. [CrossRef]

28. Vaidman, L. Comment on "Particle path through a nested Mach-Zehnder interferometer". Phys. Rev. A 2017, 95, 066101. [CrossRef] 
29. Dajka, J. Faint trace of a particle in a noisy Vaidman three-path interferometer. Sci. Rep. 2021, 11, 1123. [CrossRef] [PubMed]

30. Griffiths, R.B. Consistent quantum measurements. Stud. Hist. Philos. Sci. Part B Stud. Hist. Philos. Mod. Phys. 2015, 52, 188-197. [CrossRef]

31. Griffiths, R.B. A consistent quantum ontology. Stud. Hist. Philos. Sci. Part B Stud. Hist. Philos. Mod. Phys. 2013, 44, 93-114. [CrossRef]

32. Griffiths, R.B. The New Quantum Logic. Found. Phys. 2014, 44, 610-640. [CrossRef]

33. Griffiths, R.B. What quantum measurements measure. Phys. Rev. A 2017, 96, 032110. [CrossRef]

34. Riedel, C.J.; Zurek, W.H.; Zwolak, M. Objective past of a quantum universe: Redundant records of consistent histories. Phys. Rev. A 2016, 93, 032126. [CrossRef]

35. Craig, D.A. The consistent histories approach to loop quantum cosmology. Int. J. Mod. Phys. D 2016, 25, 1642009. [CrossRef]

36. Birkhoff, G.; Von Neumann, J. The Logic of Quantum Mechanics. Ann. Math. 1936, 37, 823-843. [CrossRef]

37. Griffiths, R.B. Consistent histories and quantum reasoning. Phys. Rev. A 1996, 54, 2759-2774. [CrossRef] [PubMed]

38. Aharmim, B.; Ahmed, S.N.; Anthony, A.E.; Beier, E.W.; Bellerive, A.; Bergevin, M.; Biller, S.D.; Boger, J.; Boulay, M.G.; Bowler, M.G.; et al. Electron energy spectra, fluxes, and day-night asymmetries of ${ }^{8} \mathrm{~B}$ solar neutrinos from measurements with $\mathrm{NaCl}$ dissolved in the heavy-water detector at the Sudbury Neutrino Observatory. Phys. Rev. C 2005, 72, 055502. [CrossRef]

39. Giunti, C. No effect of Majorana phases in neutrino oscillations. Phys. Lett. B 2010, 686, 41-43. [CrossRef] 